Pacific Journal of Mathematics

SEPARATIVE RELATIONS FOR MEASURES

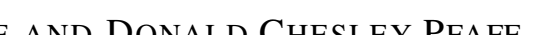




\title{
SEPARATIVE RELATIONS FOR MEASURES
}

\author{
A. P. Morse and D. C. Pfaff
}

When dealing with Carathéodory (outer) measures, a natural problem arises: how does one determine a nontrivial, interesting family of measurable sets? In particular cases of a metric or topological nature, it has been customary to assume that the measure is additive on sets which are a bit more than merely disjoint. The general approach of this paper, purely set-theoretical in nature, emphasizes a relation $R$ which "separates" sets, and describes certain sets, constructed with the aid of $R$, which turn out to be measurable whenever the measure is additive on sets which are separatively related.

We present several applications, some of which have appeared in the literature and others which have not, to indicate the scope of our methods.

In $\S 2$ we assemble some definitions, notations, and elementary measure-theoretic results. In $\S 3$ we define separative relations, describe certain families of sets associated with them, and proceed to prove measurability of the sets in these families, assuming that a measure is additive on sets which are separatively related. $\S 4$ is devoted to applications.

The authors express their thanks to the referee for several suggestions which have simplified and shortened this paper considerably.

2. Preliminary definitions, notations, and theorems.

DEFINITIONS 2.1.

1. $A \operatorname{cs} B=\{x: x \in A$ and $x \notin B\}$

2. $\sigma F=\bigcup_{f \in F} f=\{x: x \in f$ for some $f \in F\}$

3. $\operatorname{sb} A=\{x: x \subset A\}$

4. $\operatorname{sp} A=\{x: x \supset A\}$

DEFINITIONS 2.2.

1. $\operatorname{dmn} f=\{x:(x, y) \in f$ for some $y\}$

2. $\mathrm{dmn}^{\prime} f=\{x: x \in \mathrm{dmn} f$ and $|f(x)|<\infty\}$

3. $\operatorname{rlm} f=\sigma \mathrm{dmn} f$

4. $\operatorname{rng} f=\{y:(x, y) \in f$ for some $x\}$

DeFinITION 2.3.

$\omega$ is the set of nonnegative integers.

We shall assume that the integer 0 and the empty set are the same. Moreover, we assume that for each $n \in \omega, n=\{k \in \omega: k<n\}$. 
DEFINITIONS 2.4.

1. $\varphi$ measures $\mathscr{S}$ if and only if $\varphi$ is such a function that $\operatorname{dmn} \varphi=$ sb $\mathscr{S}, 0 \leqq \varphi(A)$ whenever $A \subset \mathscr{S}$, and

$$
\varphi(A) \leqq \sum_{\beta \in H^{\prime}} \varphi(\beta)
$$

whenever $F$ is a countable family for which

$$
A \subset \sigma F \subset \mathscr{S} \text {. }
$$

2. $\operatorname{Msr} \mathscr{S}=\{\varphi: \varphi$ measures $\mathscr{S}\}$

3. $A$ is $\varphi$-measurable if and only if $A \in \mathrm{dmn} \varphi$ and for all $T \in$ $\operatorname{dmn} \varphi$

$$
\varphi(T)=\varphi(T \cap A)+\varphi(T \propto A) .
$$

4. $\operatorname{mbl} \varphi=\{A: A$ is $\varphi$-measurable $\}$

5. sct $\varphi T$ is the function $\psi$ on $\operatorname{dmn} \varphi$ such that, for each $A \in$ $\operatorname{dmn} \varphi$,

$$
\psi(A)=\varphi(T \cap A)
$$

6. $\operatorname{sms} \varphi=\left\{\psi: \psi=\operatorname{sct} \varphi T\right.$ for some $\left.T \in \mathrm{dmn}^{\prime} \varphi\right\}$

The following Theorems 2.5 and 2.6 are fairly well-known and rather easy to prove.

Theorems 2.5 .

1. If $\varphi \in \operatorname{Msr} \mathscr{S}$, then $\operatorname{sms} \varphi \subset \operatorname{Msr} \mathscr{S}$.

2. If $\varphi \in \operatorname{Msr} \mathscr{S}$, then $A \in \operatorname{mbl} \varphi$ if and only if

$$
\psi(\mathscr{S})=\psi(A)+\psi(\mathscr{S} \odot A)
$$

for each $\psi \in \operatorname{sms} \varphi$.

3. If $\varphi \in \operatorname{Msr} \mathscr{S}, \mathscr{S}^{\prime} \subset \mathscr{S}$, and $\varphi\left(\mathscr{S} \odot \mathscr{S}^{\prime}\right)=0$, then

$$
\varphi(\mathscr{S} \cap A)=\varphi\left(\mathscr{S}^{\prime} \cap A\right)
$$

for each $A$.

Our proof of Theorem 2.6.4 has been considerably simplified by suggestions made by M. Sion and L. B. Davis.

THEOREMS 2.6.

1. If $\psi \in \operatorname{Msr} \mathscr{S}, B \subset \mathscr{S}, K_{n} \subset B$ for each $n \in \omega$, and

$$
\lim _{n \rightarrow \infty} \psi\left(B \odot K_{n}\right)=0,
$$

then

$$
\lim _{n \rightarrow \infty} \psi\left(K_{n} \cap T\right)=\psi(B \cap T)
$$


for each $T \subset \mathscr{S}$.

2. If $\psi \in \operatorname{Msr} \mathscr{S}, B \subset \mathscr{S}, \lim _{n \rightarrow \infty} \psi\left(\mathscr{S} \backsim K_{n}\right)=0$, and

$$
\psi\left(K_{n} \cap B\right)+\psi\left(K_{n} \odot B\right) \leqq \psi(\mathscr{S})
$$

for each $n \in \omega$, then

$$
\psi(B)+\psi(\mathscr{S} \operatorname{cs} B \leqq \psi(\mathscr{S})
$$

3. If $\psi \in \operatorname{Msr} \mathscr{S}, K$ is an increasing sequence of subsets of $\mathscr{S}$, $\psi\left(S \propto \bigcup_{n \in \omega} K_{n}\right)=0$, and

$$
\sum_{n \in \omega} \psi\left(K_{n+1} \curvearrowright K_{n}\right)<\infty
$$

then

$$
\lim _{n \rightarrow \infty} \psi\left(S \odot K_{n}\right)=0
$$

4. If $\psi \in \operatorname{Msr} \mathscr{S}, A$ is an increasing sequence of subsets of $\mathscr{S}$, and $\psi\left(A_{n}\right)+\psi\left(A_{n+2} \backsim A_{n+1}\right) \leqq \psi\left(A_{n+2}\right)$ for each $n \in \omega$, then

$$
\sum_{n \in \omega} \psi\left(A_{n+1} \odot A_{n}\right) \leqq 2 \cdot \psi(\mathscr{S})
$$

Proof. By induction on $N$ we find that if $N \in \omega$, then

$$
\sum_{n \in N+1} \psi\left(A_{n+1} \backsim A_{n}\right) \leqq \psi\left(A_{N}\right)+\psi\left(A_{N+1}\right) \text {. }
$$

Letting $N$ tend to infinity completes the proof.

The following alternate annular ring principle is a variant of a theorem given by A. P. Morse in his 1958-1959 lectures on Real Variable at the University of California.

THEOREM 2.7. If $\psi \in \mathrm{Msr} \mathscr{S}, A$ is an increasing sequence of subsets of $\mathscr{S}, C \cup \mathrm{U}_{n \in \omega} A_{n}=\mathscr{S}^{\prime} \subset \mathscr{S}, \psi\left(\mathscr{S} \backsim \mathscr{S}^{\prime}\right)=0$, and $\psi\left(A_{n}\right)+$ $\psi\left(A_{n+2} \backsim A_{n+1}\right) \leqq \psi\left(A_{n+2}\right)$ and $\psi\left(A_{n}\right)+\psi(C) \leqq \psi(\mathscr{S})$ for each $n \in \omega$, then

$$
\psi(C)+\psi(\mathscr{S} \backsim C) \leqq \psi(\mathscr{S})
$$

Proof. Since the conclusion is obvious if $\psi(\mathscr{S})=\infty$, we henceforth assume $\psi(\mathscr{S})<\infty$. Now we let

$$
\begin{aligned}
B & =\bigcup_{n \in \omega} A_{n}, \\
K_{n} & =C \cup A_{n} \text { for each } n \in \omega,
\end{aligned}
$$

and complete the proof in Step IV below.

Step I. $\lim _{n \rightarrow \infty} \psi\left(B \backsim A_{n}\right)=0$. 
Proof. Since we are assuming $\psi(\mathscr{S})<\infty$, Theorem 2.6.4 assures us that $\sum_{n \in \omega} \psi\left(A_{n+1} \propto A_{n}\right)<\infty$, and thus by Theorem 2.6.3 we have

$$
\lim _{n \rightarrow \infty} \psi\left(B \backsim A_{n}\right)=0 \text {. }
$$

Step II. $\lim _{n \rightarrow \infty} \psi\left(\mathscr{S} \propto K_{n}\right)=0$.

Proof. Using Theorem 2.5.3 and the obvious fact that $B \subset C=$ $\mathscr{S}^{\prime} \operatorname{cs} C$, we see that, for each $n \in \omega$,

$$
\begin{aligned}
& \psi\left(\mathscr{S} \circlearrowleft K_{n}\right)=\psi\left(\mathscr{S} \cos \left(C \cup A_{n}\right)\right) \\
& =\psi\left((\mathscr{S} \odot C) \propto A_{n}\right) \\
& =\psi\left(\left(\mathscr{S}^{\prime} \backsim C\right) \propto A_{n}\right) \\
& =\psi\left((B ⿻ s) \propto A_{n}\right) \\
& \leqq \psi\left(B \backsim A_{n}\right) \text {. }
\end{aligned}
$$

Hence, by Step I, $\lim _{n \rightarrow \infty} \psi\left(\mathscr{S} \backsim K_{n}\right)=0$.

Step III. For each $n \in \omega$,

$$
\psi\left(K_{n} \cap C\right)+\psi\left(K_{n} \propto C\right) \leqq \psi(\mathscr{S}) .
$$

Proof. If $n \in \omega$, then

$$
\begin{aligned}
\psi\left(K_{n} \cap C\right)+\psi\left(K_{n} \propto C\right) & =\psi\left(\left(C \cup A_{n}\right) \cap C\right)+\psi\left(\left(C \cup A_{n}\right) \propto \dot{C}\right) \\
& =\psi(C)+\psi\left(A_{n} \odot C\right) \\
& \leqq \psi(C)+\psi\left(A_{n}\right) \\
& \leqq \psi(\mathscr{S}) .
\end{aligned}
$$

Step IV. $\psi(C)+\psi(\mathscr{S}$ c $C) \leqq \psi(\mathscr{S})$.

Proof. Use Step II, Step III, and Theorem 2.6.2.

3. Separative relations. We now describe the objects of main interest in this paper.

DEFINITIONS 3.1.

1. preseparative $=\{R: R$ is a relation, $\operatorname{rlm} R=\sigma \operatorname{rng} R, R \neq 0$, and whenever

$$
(A, B) \in R,(C, B) \in R, A^{\prime} \subset A, B^{\prime} \subset B,
$$

it follows that

$$
\left.(A \cup C, B) \in R \text { and }\left(A^{\prime}, B^{\prime}\right) \in R\right\} \text {. }
$$


2. separative $=\{R \in$ preseparative: whenever $(A, B) \in R$, there exists $A^{\prime} \in \operatorname{sp} A$ such that

$$
\left(A^{\prime}, B\right) \in R \quad \text { and } \quad\left(A, \alpha \odot A^{\prime}\right) \in R
$$

for each $\alpha \in \mathrm{dmn} R$.

3. Separative $=\{R \in$ preseparative: whenever $(A, B) \in R$ and $\alpha \in$ $\operatorname{dmn} R$, there exists $A^{\prime} \in \operatorname{sp} A$ such that

$$
\left.\left(\alpha \cap A^{\prime}, \alpha \cap B\right) \in R \quad \text { and } \quad\left(A, \alpha \odot A^{\prime}\right) \in R\right\} .
$$

4. shut $R=\{C$ : there exists a countable family $G$ such that

$$
\sigma \operatorname{rng} R=C \cup \sigma G \quad \text { and } \quad(g, C) \in R
$$

for each $g \in G\}$.

5. Shut $R=\{C$ : for every $\alpha \in \mathrm{dmn} R$, there exists a countable family $G$ such that

$$
\alpha=(\alpha \cap C) \cup \sigma G \quad \text { and } \quad(g, \alpha \cap C) \in R
$$

for each $g \in G\}$.

\section{DeFinitions 3.2.}

1. adt $R=\{\varphi \in \operatorname{Msr} \sigma \operatorname{rng} R: \varphi(A \cup B)=\varphi(A)+\varphi(B)$ whenever $(A, B) \in R\}$.

2. Adt $R=\left\{\varphi \in\right.$ adt $R$ : for every $T \in \mathrm{dmn}^{\prime} \varphi$, there exists such a countable family $G$ of elements of $\operatorname{dmn} R$ that $\varphi(T \backsim \sigma G)=0\}$.

3. Sepad $R=\left\{\varphi \in\right.$ adt $R$ : for every $T \in \mathrm{dmn}^{\prime} \varphi$, there exists an increasing sequence $K$ of elements of $\operatorname{dmn} R \cap$ Shut $R$ such that $\left.\varphi\left(T \propto \bigcup_{n \in \omega} K_{n}\right)=0\right\}$.

Theorems 3.3, 3.4, and 3.5 below are easily verified.

\section{THEOREMS 3.3.}

1. separative $\subset$ Separative $\subset$ preseparative.

2. If $R \in$ Separative and $\operatorname{rm} R \in \operatorname{dmn} R$, then $R \in$ separative.

\section{THEOREMS 3.4.}

1. If $R \in$ preseparative, then shut $R \subset$ Shut $R$.

2. If $R \in$ preseparative and $\operatorname{rm} R \in \mathrm{dmn} R$, then shut $R=$ Shut $R$.

3. If $R \in$ preseparative, then $0 \in$ Shut $R$ and $\operatorname{rlm} R \in$ Shut $R$.

\section{THEOREMS 3.5.}

1. Sepad $R \subset$ Adt $R \subset$ adt $R$.

2. If $R \in$ preseparative and $\operatorname{rlm} R \in \operatorname{dmn} R$, then adt $R=\operatorname{Adt} R=$ Sepad $R$. 
THEOREMS 3.6.

1. If $R \in$ preseparative, $\varphi \in \operatorname{adt} R$ and $\psi \in \operatorname{sms} \varphi$, then $\psi \in \operatorname{adt} R$.

Proof. Let $\psi=\operatorname{sct} \varphi T$. Using 3.1.1, we see that if $(A, B) \in R$, then $(T \cap A, T \cap B) \in R$, and hence

$$
\varphi((T \cap A) \cup(T \cap B))=\varphi(T \cap A)+\varphi(T \cap B) .
$$

Thus $\psi(A \cup B)=\psi(A)+\psi(B)$.

2. If $R \in$ preseparative, $\varphi \in$ Adt $R$, and $\psi \in \operatorname{sms} \varphi$, then $\psi \in \operatorname{Adt} R$.

Proof. Clearly $\psi \in \operatorname{adt} R$, so suppose $\psi=\operatorname{sct} \varphi S, T \in \mathrm{dmn}^{\prime} \psi$, and $\varphi(S)<\infty$. There is such a countable family $G$ of elements of $\mathrm{dmn} R$ that $\varphi(S \propto \sigma G)=0$. Hence $\varphi(S \cap(T \propto \sigma G))=0$, which implies that $\psi(T \backsim \sigma G)=0$.

3. If $R \in$ preseparative, $\varphi \in \operatorname{Sepad} R$, and $\psi \in \operatorname{sms} \varphi$, then $\psi \in$ Sepad $R$.

The proof of 3.6.3 is similar to that of 3.6.2.

THEOREM 3.7. If $R \in$ separative and $\left(A_{n}, C\right) \in R$ for each $n \in \omega$, then there exists an increasing sequence $A^{\prime}$ such that, for every $n \in \omega$,

$$
\left(A_{n}^{\prime}, A_{n+2}^{\prime} \text { c } A_{n+1}^{\prime}\right) \in R,\left(A_{n}^{\prime}, C\right) \in R \text {, and } A_{n} \subset A_{n}^{\prime} \text {. }
$$

Proof. Repeatedly use Definition 3.1.2 to determine inductively such a sequence $A^{\prime}$ that for each $\alpha \in \operatorname{dmn} R$ and $n \in \omega$ we have

$$
\begin{aligned}
& A_{0} \subset A_{0}^{\prime},\left(A_{0}^{\prime}, C\right) \in R,\left(A_{0}, \alpha \backsim A_{0}^{\prime}\right) \in R, \\
& A_{n+1} \cup A_{n}^{\prime} \subset A_{n+1}^{\prime},\left(A_{n+1}^{\prime}, C\right) \in R,
\end{aligned}
$$

and

$$
\left(A_{n+1} \cup A_{n}^{\prime}, \alpha \odot A_{n+1}^{\prime}\right) \in R \text {. }
$$

Clearly $A_{n}^{\prime} \in \mathrm{dmn} R$ for each $n \in \omega$. Hence, using Definitions 3.1.1 and 3.1 .2 we see that for each $n \in \omega$,

$$
\left(A_{n}^{\prime}, A_{n+2}^{\prime} \odot A_{n+1}^{\prime}\right) \in R .
$$

The remaining conclusions are obvious.

Our next theorem is a direct generalization of a well-known theorem of Carathéodory [2].

THEOREM 3.8. If $R \in$ separative, $C \in$ shut $R$, and $\varphi \in$ adt $R$, then $C \in \operatorname{mbl} \varphi$.

Proof. Let $\mathscr{S}=\operatorname{rlm} R$ and $\psi \in \operatorname{sms} \varphi$. Use Definition 3.1.4 to find such a sequence $A$ that, for each $n \in \omega$, 


$$
\left(A_{n}, C\right) \in R \text { and } \bigcup_{n \in \omega} A_{n} \cup C=\mathscr{S} \text {. }
$$

Now use Theorem 3.7 to determine such a sequence $A^{\prime}$ that, for each $n \in \omega$,

$$
A_{n} \subset A_{n}^{\prime} \subset A_{n+1}^{\prime},\left(A_{n}^{\prime}, C\right) \in R,
$$

and

$$
\left(A_{n}^{\prime}, A_{n+2}^{\prime} \backsim A_{n+1}^{\prime}\right) \in R \text {. }
$$

From the above, and the fact that $\psi \in \operatorname{adt} R$, we see the following:

(1) $A^{\prime}$ is an increasing sequence of subsets of $\mathscr{S}$.

(2) $C \cup \cup_{n \in \omega} A_{n}^{\prime}=\mathscr{S}$.

(3) $\psi\left(\mathscr{S} \operatorname{cs}\left(C \cup \cup_{n \in \omega} A_{n}^{\prime}\right)\right)=0$.

(4) $\psi\left(A_{n+2}^{\prime}\right) \geqq \psi\left(A_{n}^{\prime} \cup\left(A_{n+2}^{\prime}\right.\right.$ ⿻s $\left.\left.A_{n+1}^{\prime}\right)\right)=\psi\left(A_{n}^{\prime}\right)+\psi\left(A_{n+2}^{\prime} \infty A_{n+1}^{\prime}\right)$ for each $n \in \omega$.

(5) $\psi(\mathscr{S}) \geqq \psi\left(A_{n}^{\prime} \cup C\right)=\psi\left(A_{n}^{\prime}\right)+\psi(C)$, for each $n \in \omega$. Referring to Theorem 2.7, we infer

$$
\psi(C)+\psi(\mathscr{S} \odot C) \leqq \psi(\mathscr{S}) \text {. }
$$

The reverse inequality is obvious. Hence, since $\psi$ is an arbitrary member of $\operatorname{sms} \varphi$, we see from Theorem 2.5.2 that $C \in \operatorname{mbl} \varphi$.

We pave the way towards our next major measurability theorem by proving two preliminary theorems and stating a result of Trevor J. McMinn [4].

Theorem 3.9. If $R \in$ Separative, $C \in$ Shut $R, \alpha \in \operatorname{dmn} R, \varnothing \in \operatorname{adt} R$, $\psi=\operatorname{sct} \varphi \alpha$, and $\mathscr{S}=\operatorname{rlm} R$, then

$$
\psi(C)+\psi(\mathscr{S} \odot C) \leqq \psi(\mathscr{S}) \text {. }
$$

Proof. Use Definition 3.1.5 to find such a sequence $A$ that, for every $n \in \omega,\left(A_{n}, \alpha \cap C\right) \in R$, and $\alpha=(\alpha \cap C) \cup \cup_{n \in \omega} A_{n}$. Now use Definition 3.1.3 to determine inductively such a sequence $A^{\prime}$ that for each $n \in \omega$,

$$
\begin{aligned}
& A_{0}^{\prime} \supset A_{0},\left(\alpha \cap A_{0}^{\prime}, \alpha \cap C\right) \in R,\left(A_{0}, \alpha \backsim A_{0}^{\prime}\right) \in R, \\
& A_{n+1}^{\prime} \supset\left(\alpha \cap A_{n}^{\prime}\right) \cup A_{n+1},\left(\alpha \cap A_{n+1}^{\prime}, \alpha \cap C\right) \in R,
\end{aligned}
$$

and

$$
\left(\left(\alpha \cap A_{n}^{\prime}\right) \cup A_{n+1}, \alpha \propto A_{n+1}^{\prime}\right) \in R .
$$

For each $n \in \omega$, let $K_{n}=\alpha \cap A_{n}^{\prime}$. We now divide the remainder of the proof into six steps, the first of which is obvious.

Step I. For each $n \in \omega, K_{n} \subset K_{n+1}$.

Step II. $\psi\left(\mathscr{S}\right.$ cs $\left.\left((\alpha \cap C) \cup \bigcup_{n \in \omega} K_{n}\right)\right)=0$. 
Proof. Since $A_{n}^{\prime} \supset A_{n}$ for each $n \in \omega$, it follows that

$$
(\alpha \cap C) \cup \bigcup_{n \in \omega} A_{n}^{\prime} \supset(\alpha \cap C) \cup \bigcup_{n \in \omega} A_{n}=\alpha,
$$

and hence $C \cup \cup_{n \in \omega} A_{n}^{\prime} \supset \alpha$. Therefore,

$$
\begin{aligned}
& \psi\left(\mathscr{S} \operatorname{cs}\left((\alpha \cap C) \cup \bigcup_{n \in \omega} K_{n}\right)\right) \\
= & \psi\left(\alpha \operatorname{cs}\left((\alpha \cap C) \cup \bigcup_{n \in \omega}\left(\alpha \cap A_{n}^{\prime}\right)\right)\right) \\
= & \psi\left(\alpha \operatorname{cs}\left(\alpha \cap\left(C \cup \bigcup_{n \in \omega} A_{n}^{\prime}\right)\right)\right) \\
= & \psi\left(\alpha \operatorname{cs}\left(C \cup \bigcup_{n \in \omega} A_{n}^{\prime}\right)\right) \\
= & \psi(0) \\
= & 0 .
\end{aligned}
$$

Step III. For each $n \in \omega$,

$$
\psi\left(K_{n}\right)+\psi\left(K_{n+2} \odot K_{n+1}\right) \leqq \psi\left(K_{n+2}\right) .
$$

Proof. If $n \in \omega$, then

$$
\begin{aligned}
& \psi\left(K_{n}\right)+\psi\left(K_{n+2} \propto K_{n+1}\right) \\
& =\psi\left(\alpha \cap A_{n}^{\prime}\right)+\psi\left(\left(\alpha \cap A_{n+2}^{\prime}\right) \propto s\left(\alpha \cap A_{n+1}^{\prime}\right)\right) \\
& =\psi\left(\alpha \cap A_{n}^{\prime}\right)+\psi\left(\left(\alpha \cap A_{n+2}^{\prime}\right) \cap\left((\mathscr{S} \operatorname{cs} \alpha) \cup\left(\mathscr{S} \operatorname{cs} A_{n+1}^{\prime}\right)\right)\right) \\
& =\psi\left(\alpha \cap A_{n}^{\prime}\right)+\psi\left(\left(\alpha \cap A_{n+2}^{\prime}\right) \propto A_{n+1}^{\prime}\right) \\
& =\psi\left(\left(\alpha \cap A_{n}^{\prime}\right) \cup\left(\left(\alpha \cap A_{n+2}^{\prime}\right) \propto A_{n+1}^{\prime}\right)\right) \\
& =\psi\left(K_{n} \cup\left(K_{n+2} \bowtie A_{n+1}^{\prime}\right)\right) \\
& \leqq \psi\left(K_{n+2}\right) \text {. }
\end{aligned}
$$

Step IV. For each $n \in \omega, \psi\left(K_{n}\right)+\psi(\alpha \cap C) \leqq \psi(\mathscr{S})$.

Proof. If $n \in \omega$, then

$$
\begin{aligned}
\psi\left(K_{n}\right)+\psi(\alpha \cap C) & =\psi\left(\alpha \cap A_{n}^{\prime}\right)+\psi(\alpha \cap C) \\
& =\psi\left(\left(\alpha \cap A_{n}^{\prime}\right) \cup(\alpha \cap C)\right) \\
& \leqq \psi(\mathscr{S}) .
\end{aligned}
$$

Step V. $\psi(\alpha \cap C)+\psi(\mathscr{S} \propto(\alpha \cap C)) \leqq \psi(\mathscr{S})$.

Proof. Use Theorem 2.7, and Steps I, II, III, and IV.

Step VI. $\psi(C)+\psi(\mathscr{S} \backsim C) \leqq \psi(\mathscr{S})$.

Proof. 


$$
\begin{aligned}
& \psi(C)+\psi(\mathscr{S} \backsim C) \\
= & \psi(\alpha \cap C)+\psi(\mathscr{S} \odot C) \\
\leqq & \psi(\alpha \cap C)+\psi((\mathscr{S} \propto \alpha) \cup(\mathscr{S} \odot C)) \\
= & \psi(\alpha \cap C)+\psi(\mathscr{S} \odot(\alpha \cap C)) \\
\leqq & \psi(\mathscr{S}) .
\end{aligned}
$$

Theorem 3.10. If $R \in$ Separative, $C \in$ Shut $R, \alpha \in \mathrm{dmn} R, \varphi \in$ adt $R$, and $\psi=\operatorname{set} \varphi \alpha$, then $C \in \operatorname{mbl} \psi$.

Proof. Suppose $\mathscr{S}=\operatorname{rlm} R, \theta \in \operatorname{sms} \psi$, and $\theta=\operatorname{sct} \psi T$. Then $\theta=\operatorname{sct} \varphi(T \cap \alpha)$. But since $T \cap \alpha \subset \alpha, T \cap \alpha \in \mathrm{dmn} R$. Thus by the preceding theorem we have

$$
\theta(C)+\theta(\mathscr{S} \propto C) \leqq \theta(\mathscr{S}) .
$$

The reverse inequality is obvious, so by Theorem 2.5.2 we have $C \in$ $\mathrm{mbl} \psi$.

THEOREM 3.11. (McMinn). Suppose $\varphi \in$ Msr $\mathscr{S}$, and for every $T$ for which $\varphi(T)<\infty$ there exists a sequence $k$ such that

$$
\varphi\left(T \backsim \bigcup_{n \in \omega} k_{n}\right)=0
$$

and

$k_{n} \subset k_{n+1} \subset \mathscr{S}, A \in \mathrm{mbl} \operatorname{set} \varphi k_{n}$, and $k_{n} \in \operatorname{mbl} \operatorname{sct} \varphi k_{n+1}$ for each $n \in \omega$. Then $A \in \operatorname{mbl} \varphi$.

TheOREm 3.12. If $R \in$ Separative, $C \in \operatorname{Shut} R$, and $\varphi \in \operatorname{Sepad} R$, then $C \in \operatorname{mbl} \varphi$.

Proof. Let $\mathscr{S}=\operatorname{rlm} R$. Clearly $\varphi \in \operatorname{Msr} \mathscr{S}$. Suppose $\psi \in \operatorname{sms} \varphi$ and $\psi=\operatorname{sct} \varphi T$ with $\varphi(T)<\infty$. Then by Definition 3.2.3 there is an increasing sequence $K$ of members of $\operatorname{dmn} R \cap$ Shut $R$ such that

$$
\varphi\left(T \backsim \bigcup_{n \in \omega} K_{n}\right)=0 .
$$

Hence, by Theorem 3.10 we have, for each $n \in \omega$,

$$
C \in \mathrm{mbl} \operatorname{set} \varphi K_{n} \text { and } K_{n} \in \mathrm{mbl} \operatorname{set} \varphi K_{n+1} \text {. }
$$

Theorem 3.11 now assures us that $C \in \mathrm{mbl} \varphi$.

With the help of Theorem 3.3.1, we see that Theorems 3.13 and 3.14 below follow from Theorems 3.9 and 3.10, respectively. Although they are not of intrinsic interest, they are useful in establishing our 
last measurability result.

Theorem 3.13. If $R \in$ separative, $\varphi \in \operatorname{adt} R, T \in \mathrm{dmn} R, \psi=\operatorname{sct} \varphi T$, $\mathscr{S}=\operatorname{rlm} R$, and $C \in$ Shut $R$, then $\psi(C)+\psi(\mathscr{S}$ cs $C) \leqq \psi(\mathscr{S})$.

THEOREM 3.14. If $R \in$ separative, $\varphi \in \operatorname{adt} R, T \in \operatorname{dmn} R, \psi=\operatorname{set} \varphi T$, and $C \in$ Shut $R$, then $C \in \mathrm{mbl} \psi$.

THEOREM 3.15. If $R \in$ separative, $\varphi \in \operatorname{Adt} R$, and $C \in$ Shut $R$, then $C \in \operatorname{mbl} \varphi$.

Proof. Let $\mathscr{S}=\operatorname{rlm} R$ and $\psi \in \operatorname{sms} \varphi$. Since Theorem 3.6.2 assures us that $\psi \in \operatorname{Adt} R$, and since $\psi(\mathscr{S})<\infty$, we may select a sequence $D$ of elements of $\operatorname{dmn} R$ such that $\psi\left(\mathscr{S}\right.$ \& $\left.\bigcup_{n \in \omega} D_{n}\right)=0$. Using Theorem 3.7, find a sequence $D^{\prime}$ such that, for each $n \in \omega$,

$$
\left(D_{n}^{\prime}, 0\right) \in R,\left(D_{n}^{\prime}, D_{n+2}^{\prime} \odot D_{n+1}^{\prime}\right) \in R,
$$

and

$$
D_{n} \subset D_{n}^{\prime} \subset D_{n+1}^{\prime}
$$

Noting that, for each $n \in \omega$,

$$
\begin{aligned}
\psi\left(D_{n+2}^{\prime}\right) & \geqq \psi\left(D_{n}^{\prime} \cup\left(D_{n+2}^{\prime} \propto D_{n+1}^{\prime}\right)\right) \\
& =\psi\left(D_{n}^{\prime}\right)+\psi\left(D_{n+2}^{\prime} \propto D_{n+1}^{\prime}\right),
\end{aligned}
$$

we conclude from Theorem 2.6.4 that

$$
\sum_{n \in \omega} \psi\left(D_{n+1}^{\prime} \propto D_{n}^{\prime}\right)<\infty \text {. }
$$

Hence we infer from Theorem 2.6.3 that

$$
\lim _{n \rightarrow \infty} \psi\left(\mathscr{S} \propto D_{n}^{\prime}\right)=0 \text {. }
$$

The (3.14) fact that $C \in \operatorname{mbl} \operatorname{sct} \varphi D_{n}^{\prime}$ for each $n \in \omega$, together with Theorem 2.6.2, now yields

$$
\psi(C)+\psi(\mathscr{S} \operatorname{cs}) \leqq \psi(\mathscr{S})
$$

Reference to Theorem 2.5.2 completes the proof.

REMARK 3.16. In general, we cannot show that if Shut $R \subset \mathrm{mbl} \varphi$, then $\varphi \in$ adt $R$. This seems to be due partly to the fact that we did not require in the definition of a separative relation $R$ that $(A, B) \in$ $R$ implies $A \cap B=0$. If we do add this condition, we can arrive at the above result, as the next theorem shows. 
THEOREM 3.17. If $R \in$ separative, $\mathscr{S}=\operatorname{rlm} R, \varphi \in \operatorname{Msr} \mathscr{S}$, Shut $R \subset$ $\mathrm{mbl} \varphi$, and $A \cap B=0$ whenever $(A, B) \in R$, then

$$
\varphi \in \operatorname{adt} R \text {. }
$$

Proof. Suppose $(A, B) \in R$. Proceeding as in the first sentence of the proof of Theorem 3.7 with $A_{n}=A$ for each $n \in \omega$, we determine inductively such a sequence $A^{\prime}$ that, for every $\alpha \in \mathrm{dmn} R$ and every $n \in \omega$, we have

$$
\left(A_{n}^{\prime}, \alpha \odot A_{n+1}^{\prime}\right) \in R,\left(A_{n}^{\prime}, B\right) \in R, \quad \text { and } \quad A \subset A_{n}^{\prime} \subset A_{n+1}^{\prime} .
$$

Let $C^{\prime}=\bigcup_{n \in \omega} A_{n}^{\prime}$ and $C=\mathscr{S} \propto C^{\prime}$. We complete the proof in three steps, the first of which is evident.

Step I. $A \subset \mathscr{S} \subset C$ and $B \subset C$.

Step II. $C \in$ Shut $R$.

Proof. Let $T \in \operatorname{dmn} R$, and for each $n \in \omega$ let $G_{n}=A_{n}^{\prime} \cap T$. Clearly $T=(T \cap C) \cup \bigcup_{n \in \omega} G_{n}$. Moreover, since

$$
n \in \omega \text { implies } A_{n+1}^{\prime} \subset C^{\prime} \text {, }
$$

we have

$$
n \in \omega \text { implies } C \subset \mathscr{S} \propto A_{n+1}^{\prime}
$$

and hence

$$
n \in \omega \text { implies } T \cap C \subset T \backsim A_{n+1}^{\prime} \text {. }
$$

Therefore, since $\left(A_{n}^{\prime}, T \propto A_{n+1}^{\prime}\right) \in R$, we have

$$
\left(G_{n}, T \cap C\right) \in R \text {. }
$$

The desired conclusion is now at hand.

Step III. $\varphi \in$ adt $R$.

Proof. From Step I, Step II, and the assumed measurability of members of Shut $R$, we deduce that

$$
\varphi(A \cup B)=\varphi((A \cup B) \cap C)+\varphi((A \cup B) \backsim C)=\varphi(A)+\varphi(B) .
$$

Reference to Definition 3.2.1 completes the proof.

4. Applications, In this section we examine some specific separative relations and obtain measurability theorems in metric and topological settings.

We begin by showing that the classical Caratheodory theorem on measurability of closed sets in a metric space follows from our 
general results. Following this, we prove a theorem, first published by T. J. McMinn in [4], but known earlier to A. P. Morse, which states that additivity of a measure on bounded sets of positive distance apart in a metric space $\mathscr{S}$ ensures the measurability of all closed sets in $\mathscr{S}$.

Turning to some examples with a topological flavor, we establish a theorem of Bourbaki [1] as a consequence of our theory. Next we deal with applications which involve additivity of a measure on sets whose closures do not intersect and the first of which is compact. Some of these have been anticipated by A. P. Morse, but none have appeared in the literature. In particular, Theorem 4.16 extends measurability theory to regular spaces and a fairly general class of measures.

We conclude by showing how some measurability results in a recent paper by M. Sion and R. C. Willmott [5] dealing with constructed measures can be obtained from our approach.

We begin by introducing some necessary metric and topological terminology.

\section{DEFINITIONS 4.1.}

1. $\rho$ metrizes $\mathscr{S}$ if and only if $\rho$ is such a function with domain $\mathscr{S} \times \mathscr{S}$ that

$$
0=\rho(x, x) \leqq \rho(x, y) \leqq \rho(x, z)+\rho(y, z)<\infty
$$

whenever $x \in \mathscr{S}, y \in \mathscr{S}$, and $z \in \mathscr{S}$.

2. $\operatorname{sr} \rho x r=\{y: \rho(x, y) \leqq r\}$

We note in passing that a function $\rho$ of the kind referred to in 4.1.1 is often called a pseudometric.

\section{DEFINITIONS 4.2 .}

1. Fsigma $\mathscr{T}=\{B \in \mathscr{T}: \mathscr{T}$ is a topology and $B$ is a countable union of closed sets $\}$.

2. Gdelta $\mathscr{T}=\{C: \mathscr{T}$ is a topology, $C$ is closed, and $C$ is a countable intersection of elements of $\mathscr{T}\}$.

3. $\mathscr{T}$ is locally compact if and only if $\mathscr{T}$ is a topology and each element of $\sigma \mathscr{T}$ has a neighborhood whose closure is compact.

The first theorem of this section shows that we have indeed generalized the classical Carathéodory theorem on measurability of closed sets in a metric space.

THEOREM 4.3. If $\rho$ metrizes $\mathscr{S}, R=\{(A, B)$ : the distance between $A$ and $B$ is positive\}, and $\varphi \in$ adt $R$, then:

1. $R \in$ separative, 
2. each closed set is an element of shut $R$, and

3. each closed set is $\varphi$-measurable.

Proof of 1. Clearly $R \in$ preseparative, so suppose $(A, B) \in R$ and the distance between $A$ and $B$ is $r$. According to Definition 3.1.2, we must verify the existence of a set $A^{\prime} \in \operatorname{sp} A$ such that $\left(A^{\prime}, B\right) \in R$ and $\left(A, \alpha \operatorname{cs} A^{\prime}\right) \in R$, whenever $\alpha \in \operatorname{dmn} R$. If $0<r<\infty$, a routine argument shows that we may choose $A^{\prime}$ to be the set

$$
\bigcup_{x \in A} \operatorname{sr} \rho x(r / 2) \text {. }
$$

If $r=\infty$, then either $A$ or $B$ is empty, and we may take $A^{\prime}=A$.

Proof of 2. Suppose $C$ is closed. For each $n \in \omega$, let $A_{n}=\{x$ : the distance from $x$ to $C$ is greater than or equal to $1 /(n+1)$. Evidently

$$
C \cup \cup_{n \in \omega} A_{n}=\mathscr{S}
$$

and, for each $n \in \omega,\left(A_{n}, C\right) \in R$. Reference to 3.1.4 completes the proof.

Proof of 3. Use 1, 2, and Theorem 3.8.

With the help of Theorems 3.3.1, 3.4.2, and 3.5.2, one easily checks that, if $R$ is the relation given in the preceding theorem, then: $R \in$ Separative, shut $R=$ Shut $R$, and adt $R=\operatorname{Adt} R=\operatorname{Sepad} R$. Thus the conclusion 4.3.3 is also a consequence of Theorem 3.15 and of Theorem 3.12. In general, of course, the above equalities do not hold. The following theorem, discussed at the beginning of this section, provides an example in which shut $R \neq$ Shut $R$.

THEOREM 4.4. If $\rho$ metrizes $\mathscr{S}, R=\{(A, B)$ : the distance between $A$ and $B$ is positive and $A$ and $B$ are bounded $\}$, and $\varphi \in$ adt $R$, then:

1. $R \in$ separative,

2. each closed set is an element of Shut $R$,

3. $\varphi \in \operatorname{Adt} R$, and

4. each closed set is $\varphi$-measurable.

Proof of 1. The proof is similar to that of 4.3.1, so we omit it.

Proof of 2. Suppose $C$ is closed and $\alpha \in \mathrm{dmn} R$. For each $n \in \omega$, let

$A_{n}=\{x:$ the distance from $x$ to $C$ is greater than or equal to $1 /(n+1)\}$ 
and

$$
B_{n}=A_{n} \cap \alpha
$$

Then clearly

$$
\alpha=(\alpha \cap C) \cup \bigcup_{n \in \omega} B_{n}
$$

Also, $\left(B_{n}, \alpha \cap C\right) \in R$ for each $n \in \omega$. Thus Definition 3.1.5 assures us that $C \in$ Shut $R$.

Proof of 3. Let $G=\{\operatorname{sr} \rho x n: n \in \omega\}$ and refer to Definition 3.2.2.

Proof of 4. Use 1, 2, 3, and Theorem 3.15.

One verifies without difficulty that, if $R$ is the relation described in Theorem 4.4, then $R \in$ Separative and adt $R=\operatorname{Adt} R=\operatorname{Sepad} R$. Hence the conclusion 4.4.4 also follows from Theorem 3.12. Of course, shut $R$ is not equal to Shut $R$, since an element of shut $R$ must also be an element of $\mathrm{dmn} R$, and hence bounded.

After pausing to prove the Bourbaki Theorem, we shall consider other applications of a topological nature which illuminate the differences between our main concepts. In each case we will be dealing with a relation $R$ and will need to prove that $R \in$ separative or $R \in$ Separative. Since it will always be obvious that $R \in$ preseparative, we shall omit explicit mention of this fact from our proofs.

THeOREM 4.5. If $\mathscr{T}$ is a normal topology, $\mathscr{S}=\sigma \mathscr{T}, R=\{(A$, $B): \bar{A} \cap \bar{B}=0\}$, and $\varphi \in$ adt $R$, then:

1. $R \in$ separative,

2. Gdelta $\mathscr{T} \subset$ shut $R$, and

3. Gdelta $\mathscr{T} \subset \operatorname{mbl} \varphi$.

Proof of 1. Assume that $\bar{A} \cap \bar{B}=0$. Since $\mathscr{T}$ is normal, there exists a set $A^{\prime} \in \mathscr{T}$ such that

$$
\bar{A} \subset A^{\prime} \quad \text { and } \quad \bar{A}^{\prime} \subset \mathscr{S} \propto \bar{B} \text {. }
$$

Hence $\left(A^{\prime}, B\right) \in R$ and $\left(A, \mathscr{S} \backsim A^{\prime}\right) \in R$. Thus, for each $\alpha \in \operatorname{dmn} R$, $\left(A, \alpha \backsim A^{\prime}\right) \in R$. Reference to Definition 3.1.2 completes the proof.

Proof of 2. Let $C \in \mathrm{Gdelta} \mathscr{T}$, and select such a countable subfamily $G$ of $\mathscr{T}$ that $C$ is the intersection of the elements of $G$. Then

$$
\mathscr{S}=C \cup \bigcup_{g \in G}(\mathscr{S} \odot g)
$$


and, for each $g \in G$, we have $\overline{\mathscr{S} c s g} \cap \bar{C}=0$. According to Definition 3.1.4, $C \in$ shut $R$.

Proof of 3. Use 1, 2, and Theorem 3.8.

In Theorems 4.7 through 4.16 we agree that $\mathscr{T}$ is a topology, $\mathscr{S}=\sigma \mathscr{T}$, and $R$ is the relation defined as follows:

DEFINITION 4.6.

$R=\{(A, B): \bar{A} \cap \bar{B}=0$ and $\bar{A}$ is compact $\}$.

We begin by examining the relationship between $R$ and the topological structure of $\mathscr{T}$.

Theorem 4.7. If $C$ is such a closed subset of $\mathscr{S}$ that $\mathscr{S} \backsim C$ is a countable union of closed, compact sets, then $C \in$ shut $R$.

Proof. Write $\mathscr{S} \backsim C=\bigcup_{n \in \omega} A_{n}$ with each $A_{n}$ closed and compact, and apply Definition 3.1.4.

THEOREM 4.8. Gdelta $\mathscr{T} \subset$ Shut $R$.

Proof. Suppose $C \in \mathrm{G}$ delta $\mathscr{T}$, select such a sequence $A$ of elements of $\mathscr{T}$ that

$$
C=\bigcap_{n \in \omega} A_{n},
$$

and let $\alpha \in \operatorname{dmn} R$. Notice that

$$
\alpha=(\alpha \cap C) \cup \bigcup_{n \in \omega}\left(\alpha \propto A_{n}\right) \text {. }
$$

Also, for each $n \in \omega$,

$$
\overline{\alpha \backsim A_{n}} \cap \overline{\alpha \cap C} \subset \overline{\mathscr{S} \backsim A_{n}} \cap \bar{C}=0
$$

and

$$
\overline{\alpha \operatorname{cs} A_{n}} \subset \bar{\alpha} .
$$

Since $\bar{\alpha}$ is compact, so is $\overline{\alpha c s A_{n}}$ and hence $\left(\alpha \propto s A_{n}, \alpha \cap C\right) \in R$, for each $n \in \omega$. The conclusion now follows from Definition 3.1.5.

THEOREM 4.9. If $\mathscr{T}$ is either regular or normal, then $R \in$ Separative.

Proof. We shall assume $\mathscr{T}$ is regular; the reasoning is similar if $\mathscr{T}$ is normal. 
Suppose $\bar{A} \cap \bar{B}=0, \bar{A}$ is compact, and $\alpha \in \mathrm{dmn} R$. Use Theorem 10, p. 141 in [3] to find such an $A^{\prime} \in \mathscr{T}$ that $\bar{A} \subset A^{\prime}$ and $\bar{A}^{\prime} \subset \mathscr{S}$ cs $\bar{B}$. Since $\overline{\alpha \cap A^{\prime}} \cap \overline{\alpha \cap B} \subset \bar{A}^{\prime} \cap \bar{B}=0, \overline{\alpha \cap A^{\prime}} \subset \bar{\alpha}$, and $\bar{\alpha}$ is compact, we see that $\left(\alpha \cap A^{\prime}, \alpha \cap B\right) \in R$. Moreover, because

$$
\bar{A} \cap \overline{\alpha \cos } \subset \bar{A} \cap \overline{\mathscr{S} \cos A^{\prime}}=0,
$$

we have $\left(A, \alpha \backsim A^{\prime}\right) \in R$. Reference to Definition 3.1 .3 completes the proof.

THEOREM 4.10. If $\mathscr{T}$ is locally compact and either regular or normal, then $R \in$ separative.

Proof. Suppose $\bar{A} \cap \bar{B}=0$ and $\bar{A}$ is compact. We use the fact that $\mathscr{T}$ is locally compact by covering $\bar{A}$ with elements of $\mathscr{T}$ whose closures are compact and then extracting a finite subcover to find such a $U \in \mathscr{T}$ that $\bar{A} \subset U$ and $\bar{U}$ is compact. Next, we determine such a $V \in \mathscr{T}$ that $\bar{A} \subset V$ and $\bar{V} \subset \mathscr{S} \backsim \bar{B}$. Letting $A^{\prime}=U \cap V$, we see that

$$
\begin{aligned}
& A \subset \bar{A} \subset A^{\prime}, \\
& \bar{A}^{\prime} \cap \bar{B} \subset \bar{V} \cap \bar{B}=0,
\end{aligned}
$$

and

$$
\bar{A}^{\prime} \subset \vec{U} .
$$

Hence $\bar{A}^{\prime}$ is compact and $\left(A^{\prime}, B\right) \in R$.

Moreover, since $\mathscr{S} \backsim A^{\prime}$ is closed, it follows that

$$
\bar{A} \cap \overline{\alpha \backsim A^{\prime}} \subset \bar{A} \cap \overline{\mathscr{S} \backsim A^{\prime}}=0,
$$

for each $\alpha \in \mathrm{dmn} R$. Consequently $\left(A, \alpha \backsim A^{\prime}\right) \in R$ whenever $\alpha \in \mathrm{dmn} R$. A look at Definition 3.1.2 completes the proof.

We are now in a position to obtain measurability results.

THEOREM 4.11. If $\mathscr{T}$ is locally compact and normal, $\varphi \in$ adt $R$, and $B$ is an element of $\mathscr{T}$ which can be expressed as a countable union of closed, compact sets, then $B \in \mathrm{mbl} \varphi$.

Proof. According to Theorem 4.7, $\mathscr{S} \odot B \in$ shut $R$. Hence Theorems 4.10 and 3.8 assure us that $\mathscr{S} \sim B \in \mathrm{mbl} \varphi$. The desired conclusion is at hand.

THEOREM 4.12. If $\mathscr{T}$ is locally compact and regular, $\varphi \in$ adt $R$, and $B$ is an element of $\mathscr{T}$ which can be expressed as a countable unim of compact sets, then $B \in \mathrm{mbl} \varphi$. 
Proof. Suppose $B=\bigcup_{n \in \omega} A_{n}$ and $A_{n}$ is compact for each $n \in \omega$. It is easy to check that, for each $n \in \omega, \bar{A}_{n} \subset B$ and $\bar{A}_{n}$ is compact. Hence $\mathscr{S} \propto B \in$ shut $R$, and the measurability of $B$ follows.

ThEOREM 4.13. Suppose $\mathscr{T}$ is locally compact and normal, $C \in$ G delta $\mathscr{T}$, and $\varphi \in$ adt $R$. If, in addition, for each $T \in \mathrm{dm}^{\prime} \varphi$ there exists such a family $G$ of closed compact sets that $\varphi(T \backsim \sigma G)=0$, then $C \in \operatorname{mbl} \varphi$.

Proof. Theorem 4.8 assures us that $C \in$ Shut $R$. Using the additional hypothesis concerning $\varphi$, the fact that each closed compact set is in dmn $R$, and Definition 3.2.2, we see that $\varphi \in \operatorname{Adt} R$. Hence Theorems 4.10 and 3.15 ensure $C \in \mathrm{mbl} \varphi$.

THEOREM 4.14. Suppose $\mathscr{T}$ is locally compact and regular, $C \in$ G delta $\mathscr{T}$, and $\varphi \in$ adt $R$. If, in addition, for each $T \in \mathrm{dmn}^{\prime} \varphi$ there exists such a family $G$ of compact sets that $\varphi(T \backsim \sigma G)=0$, then $C \in \mathrm{mbl} \varphi$.

Proof. In a regular space, the closure of a compact set is compact. Hence, $(A, 0) \in R$ whenever $A$ is compact, so each compact set is in $\mathrm{dmn} R$. The conclusion now follows as in 4.13 .

REMARK 4.15. We observe that $R$ is not necessarily symmetric. As a matter of fact, if $R^{\prime}$ is the symmetric relation defined by

$$
R^{\prime}=\{(A, B): \bar{A} \cap \bar{B}=0 \text { and } A \text { and } B \text { are compact }\} \text {, }
$$

then Theorems 4.10 and 4.14 remain valid if $R$ is replaced by $R^{\prime}$. Thus, if a measure $\varphi$ satisfies the additional hypothesis in Theorem 4.14, one may deduce that $\mathrm{G}$ delta $\mathscr{T} \subset \mathrm{mbl} \varphi$ by checking that $\varphi$ is additive on compact sets whose closures do not intersect.

If one is unwilling to place this restriction on $\varphi$, but wishes to determine a nontrivial class of $\varphi$-measurable sets solely from the assumption that $\varphi$ is additive on sets which are separatively related, then the nonsymmetry of $R$ is essential in many cases. This is due to the fact that the only sets we are able to prove $\varphi$-measurable in such generality are the elements of shut $R$. If $R$ is symmetric, then shut $R=0$ unless $\mathscr{S}$ is sigmacompact.

The above applies also to Theorem 4.13 .

In our next theorem, we ask somewhat more of the measure than before. In partial compensation, we are enabled to drop the assumption of local compactness.

THEOREM 4.16. If $\mathscr{T}$ is regular or normal, 


$$
\varphi \in \operatorname{adt} R, C \in \mathrm{G} \text { delta } \mathscr{T},
$$

and, corresponding to each $T \in \mathrm{dmn}^{\prime} \varphi$, there exists such a family $G$ of compact elements of $\mathrm{G}$ delta $\mathscr{T}$ that $\varphi(T \backsim \sigma G)=0$, then $C \in \mathrm{mbl} \varphi$.

Proof. We already know that $R \in$ Separative and that $\mathrm{G}$ delta $\mathscr{T} \subset$ Shut $R$. In view of Theorem 3.12, we need only show that $\varphi \in \operatorname{Sepad} R$. Let $T \in \mathrm{dmn}^{\prime} \varphi$ and choose such a sequence $K$ of compact elements of Gdelta $\mathscr{T}$ that

$$
\varphi\left(T \backsim \bigcup_{n \in \omega} K_{n}\right)=0 .
$$

By taking unions if necessary, we may clearly assume without loss of generality that $K$ is an increasing sequence. Because each $K_{n}$ is closed and compact, we see that $\left(K_{n}, 0\right) \in R$ and therefore $K_{n} \in \operatorname{dmn} R$, for each $n \in \omega$. Moreover, for each $n \in \omega, K_{n} \in$ Shut $R$, so by Definition 3.2.3, $\varphi \in \operatorname{Sepad} R$.

We conclude this paper by investigating a separative relation $R$ whose definition is considerably different in character from those previously discussed. We employ $R$ to deduce two measurability theorems which were proved in a quite different manner by Sion and Willmott in [5].

The following definitions which, for simplicity, have been slightly modified, are given on pp. 276, 279, and 280 of [5].

\section{DEFINITIONS 4.17 .}

1. $\mathscr{H}$ is a filterbase if and only if $\mathscr{H}$ is a nonempty family of sets such that for every $M \in \mathscr{H}$ and $N \in \mathscr{H}$, there exists $H \in \mathscr{H}$ such that $0 \neq H \subset M \cap N$.

2. $\mathscr{\mathscr { C }}$ is a filterbase in $\mathscr{S}$ if and only if $\mathscr{\mathscr { C }}$ is a filterbase and for every $H \in \mathscr{H}, H$ is a family of subsets of $\mathscr{S}, 0 \in H$, and $\sigma H=\mathscr{S}$.

In all subsequent definitions and theorems, we shall assume the hypothesis " $\mathscr{H}$ is a filterbase in $\mathscr{S}$ " to be affixed.

\section{DEFINITIONS 4.18.}

1. If $x \in \mathscr{S}$ and $H \in \mathscr{H}$, then $H[x]=\sigma\{h \in H: x \in h\}$.

2. If $A \subset \mathscr{S}$ and $H \in \mathscr{H}$, then

$$
H[A]=\bigcup_{x \in A} H[x]=\sigma\{h \in H: h \cap A \neq 0\} .
$$

3. $\mathscr{G}=\{G \subset \mathscr{S}$ : for every $x \in G$, there exists $H \in \mathscr{H}$ for which $H[x] \subset G\}$.

4. $\mathscr{L}$ satisfies (5 II) if and only if for each $H \in \mathscr{H}$, there exist $H_{1} \in \mathscr{P}$ and $H_{2} \in \mathscr{H}$ such that $H_{1}\left[H_{2}[A]\right] \subset H[A]$, whenever $A \subset \mathscr{S}$.

5. $\mathscr{H}$ satisfies (5 III) if and only if there exists such an $H \in \mathscr{C}$ 
that $H[A] \subset B$, whenever $A$ is $\mathscr{G}$-closed, $B \in \mathscr{G}$, and $A \subset B$.

6. $\mathscr{H}$ satisfies (5 IV) if and only if there exists such a sequence $H$ of elements of $\mathscr{H}$ that, for every $N \in \mathscr{H}$, there exists $n \in \omega$, such that $H_{n} \subset N$.

Theorems 4.19 are for the most part taken directly from [5], p. 280. They are all immediate consequences of the above definitions.

\section{THEOREMS 4.19.}

1. $\mathscr{G}$ is a topology and $\sigma \mathscr{G}=\mathscr{S}$.

2. If $H \in \mathscr{H}$ and $A_{i} \subset \mathscr{S}$ for each $i \in I$, then

$$
H\left[\bigcup_{i \in I} A_{i}\right]=\bigcup_{i \in I} H\left[A_{i}\right] \text {. }
$$

3. If $H \in \mathscr{H}, K \in \mathscr{H}, A \subset \mathscr{S}$, and $H \subset K$, then $H[A] \subset K[A]$.

4. If $H \in \mathscr{H}, A \cup B \subset \mathscr{S}$, and $A \subset B$, then $H[A] \subset H[B]$.

5. If $H \in \mathscr{C}$ and $A \cup B \subset \mathscr{S}$, then $H[A] \cap B=0$ if and only if $A \cap H[B]=0$.

We now define, for future use, a certain relation.

\section{DEFINITION 4.20 .}

$$
R=\{(A, B): \text { for some } H \in \mathscr{H}, H[A] \cap B=0\} .
$$

THEOREM 4.21. $R \in$ preseparative.

Proof. In view of Theorem 4.19.5, we see that $R$ is a symmetric relation, and hence $\mathrm{dmn} R=\operatorname{rng} R$. Thus $\operatorname{rlm} R=\sigma \operatorname{rng} R$.

Now suppose $(A, B) \in R,(C, B) \in R, A^{\prime} \subset A$, and $B^{\prime} \subset B$. Let $H$ and $K$ be such elements of $\mathscr{H}$ that $H[A] \cap B=0$ and $K[C] \cap B=0$. Using the fact that $\mathscr{H}$ is a filterbase, we find such an $L \in \mathscr{H}$ that $L \subset H \cap K$. Now, using Theorems 4.19.3 and 4.19.2, we infer:

$$
L[A] \subset H[A], L[C] \subset K[C] \text {, and } L[A \cup C]=L[A] \cup L[C] \text {. }
$$

Thus $L[A \cup C] \cap B=0$ and $(A \cup C, B) \in R$.

Finally, from Theorem 4.19.4, we infer that $H\left[A^{\prime}\right] \subset H[A]$, and thus $H\left[A^{\prime}\right] \cap B=0$. Hence $H\left[A^{\prime}\right] \cap B^{\prime}=0$ and $\left(A^{\prime}, B^{\prime}\right) \in R$. Reference to Definition 3.1.1 completes the proof.

THeOREM 4.22. If $\mathscr{\mathscr { C }}$ satisfies (5 II), then $R \in$ separative.

Proof. Suppose $(A, B) \in R$, and let $H$ be such an element of $\mathscr{H}$ that $H[A] \cap B=0$. We invoke Definition 4.18 .4 once to ascertain such members $H_{1}$ and $H_{2}$ of $\mathscr{L}$ that $H_{1}\left[H_{2}[A]\right] \subset H[A]$, and again to determine elements $H_{3}$ and $H_{4}$ of $\mathscr{H}$ such that 


$$
H_{3}\left[H_{4}\left[H_{2}[A]\right]\right] \subset H_{1}\left[H_{2}[A]\right] \text {. }
$$

Let $A^{\prime}=H_{4}\left[H_{2}[A]\right]$, and note that $A^{\prime} \supset A$.

From the above inclusions, it is evident that $H_{3}\left[A^{\prime}\right] \subset H[A]$. Hence $H_{3}\left[A^{\prime}\right] \cap B=0$ and $\left(A^{\prime}, B\right) \in R$. Moreover, we see that

$$
H_{2}[A] \subset H_{4}\left[H_{2}[A]\right],
$$

and thus $H_{2}[A] \cap\left(\mathscr{S} \backsim A^{\prime}\right)=0$. By Theorem 4.21 and Definition 3.1.2, $R \in$ separative.

THEOREM 4.23. If $\mathscr{H}$ satisfies (5 II) and (5 III) and $\varphi \in \operatorname{adt} R$, then Fsigma $\mathscr{G} \subset \operatorname{mbl} \varphi$.

Proof. Suppose $B \in \mathrm{F}$ sigma $\mathscr{G}$ and let $A$ be such a sequence of $\mathscr{G}$-closed sets that $B=\bigcup_{n \in \omega} A_{n}$. By Definition 4.18.5, for each $n \in \omega$ there exists an $H \in \mathscr{H}$ such that $H\left[A_{n}\right] \subset B$. Hence $\left(A_{n}, \mathscr{S} \propto B\right) \in$ $R$, for each $n \in \omega$. Since $(\mathscr{S} \backsim B) \cup \cup_{n \in \omega} A_{n}=\mathscr{S}$, Definition 3.1.4 assures us that $\mathscr{S}$ c $B \in$ shut $R$. The desired conclusion follows from Theorems 4.22 and 3.8 .

THEOREM 4.24. If $\mathscr{H}$ satisfies (5 II) and (5 IV) and $\varphi \in \operatorname{adt} R$, then each $\mathscr{G}$-closed set is $\varphi$-measurable.

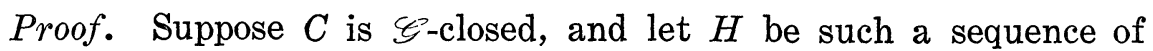
elements of $\mathscr{H}$ that, for every $N \in \mathscr{H}$, there exists $n \in \omega$ such that $H_{n} \subset N$. For each $x \in \mathscr{S} \propto C$ there clearly exists an $n \in \omega$ such that $H_{n}[x] \subset \mathscr{S} \propto C$. Let $A_{n}=\left\{x: H_{n}[x] \subset \mathscr{S} \backsim C\right\}$, for each $n \in \omega$. From Definition 4.18 .2 it follows that, for every $n \in \omega$,

$$
H_{n}\left[A_{n}\right]=\bigcup_{x \in A_{n}} H_{n}[x] \subset \mathscr{S} \subset C .
$$

Hence, for each $n \in \omega,\left(A_{n}, C\right) \in R$. Since $C \cup \cup_{n \in \omega} A_{n}=\mathscr{S}$, we have $C \in$ shut $R$. Consequently, by Theorem 3.8, $C \in \mathrm{mbl} \varphi$.

REMARK 4.25. On page 279 of [5], Sion and Willmott construct a measure $\nu$, using $\mathscr{H}$ and such a function $\tau$ on a subset $\mathscr{A}$ of $\sigma \mathscr{H}$ to the nonnegative real line that $\tau(0)=0$. They also prove that $\nu \in \operatorname{adt} R$. Thus their Theorems 7.7 and 7.8 are corollaries of our Theorems 4.23 and 4.24 .

\section{REFERENCES}

1. N. Bourbaki, Sur un théoremè de Carathéodory et la mesure dans les espaces topologiques, C. R. Acad. Sci. Paris 201 (1935), 1309-1311. 
2. C. Carathéodory, Über das lineare mass von punktmengen-eine verallgemeinerung des längenbegriffs, Nachrichten Ges. Wiss. Göttingen (1914), 404-426.

3. J. L. Kelley, General Topology, Van Nostrand, 1955.

4. T. J. McMinn, Restricted measurability, Bull. Amer. Math. Soc., 54 (1948), 1105-1109.

5. M. Sion and R. C. Willmott, Hausdorff measures on abstract spaces, Trans. Amer. Math. Soc., 123 (1966), 275-309.

Received October 15, 1970.

University of California, Berkeley

AND

University of Nevada, Reno 



\section{PACIFIC JOURNAL OF MATHEMATICS}

\section{EDITORS}

RICHARD ARENS (Managing Editor)

University of California

Los Angeles, California 90024

\author{
R. A. Beaumont \\ University of Washington \\ Seattle, Washington 98105
}

J. Dugundu*

Department of Mathematics

University of Southern California

Los Angeles, California 90007

D. Gilbarg and J. Milgram

Stanford University

Stanford, California 94305

\section{ASSOCIATE EDITORS}
E. F. BECKENBACH
B. H. NeumanN
F. WOLF
K. YosHIDA

\section{SUPPORTING INSTITUTIONS}

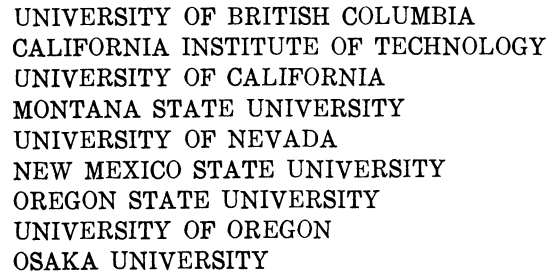

UNIVERSITY OF BRITISH COLUMBIA CALIFORNIA INSTITUTE OF TECHNOLOGY

UNIVERSITY OF CALIFORNIA

MONTANA STATE UNIVERSITY

UNIVERSITY OF NEVADA

NEW MEXICO STATE UNIVERSITY

OREGON STATE UNIVERSITY

UNIVERSITY OF OREGON

OSAKA UNIVERSITY

\author{
UNIVERSITY OF SOUTHERN CALIFORNIA \\ STANFORD UNIVERSITY \\ UNIVERSITY OF TOKYO \\ UNIVERSITY OF UTAH \\ WASHINGTON STATE UNIVERSITY \\ UNIVERSITY OF WASHINGTON \\ * * * \\ AMERICAN MATHEMATICAL SOCIETY \\ NAVAL WEAPONS CENTER
}

The Supporting Institutions listed above contribute to the cost of publication of this Journal, but they are not owners or publishers and have no responsibility for its content or policies.

Mathematical papers intended for publication in the Pacific Journal of Mathematics should be in typed form or offset-reproduced, (not dittoed), double spaced with large margins. Underline Greek letters in red, German in green, and script in blue. The first paragraph or two must be capable of being used separately as a synopsis of the entire paper. Items of the bibliography should not be cited there unless absolutely necessary, in which case they must be identified by author and Journal, rather than by item number. Manuscripts, in duplicate if possible, may be sent to any one of the four editors. Please classify according to the scheme of Math. Rev. Index to Vol. 39. All other communications to the editors should be addressed to the managing editor, or Elaine Barth, University of California, Los Angeles, California, 90024.

50 reprints are provided free for each article; additional copies may be obtained at cost in multiples of 50 .

The Pacific Journal of Mathematics is issued monthly as of January 1966. Regular subscription rate: $\$ 48.00$ a year (6 Vols., 12 issues). Special rate: $\$ 24.00$ a year to individual members of supporting institutions.

Subscriptions, orders for back numbers, and changes of address should be sent to Pacific Journal of Mathematics, 103 Highland Boulevard, Berkeley, California, 94708.

PUBLISHED BY PACIFIC JOURNAL OF MATHEMATICS, A NON-PROFIT CORPORATION

Printed at Kokusai Bunken Insatsusha (International Academic Printing Co., Ltd.), 270, 3-chome Totsuka-cho. Shinjuku-ku, Tokyo 160, Japan.

* C. R. DePrima California Institute of Technology, Pasadena, CA 91109, will replace J. Dugundji until August 1974.

Copyright (C) 1973 by

Pacific Journal of Mathematics

All Rights Reserved 


\section{Pacific Journal of Mathematics}

Vol. 48, No. $2 \quad$ April, 1973

Mir Maswood Ali, Content of the frustum of a simplex................

Mieczyslaw Altman, Contractors, approximate identities and factorization

in Banach algebras ................................ 323

Charles Francis Amelin, A numerical range for two linear operators ...... 335

John Robert Baxter and Rafael Van Severen Chacon, Nonlinear functionals

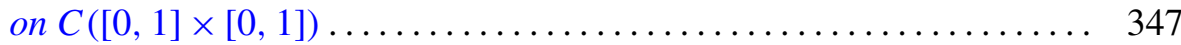

Stephen Dale Bronn, Cotorsion theories....................... 355

Peter A. Fowler, Capacity theory in Banach spaces............... 365

Jerome A. Goldstein, Groups of isometries on Orlicz spaces ........... 387

Kenneth R. Goodearl, Idealizers and nonsingular rings . ............ 395

Robert L. Griess, Jr., Automorphisms of extra special groups and

nonvanishing degree 2 cohomology ..................... 403

Paul M. Krajkiewicz, The Picard theorem for multianalytic functions . . . . 423

Peter A. McCoy, Value distribution of linear combinations of axisymmetric harmonic polynomials and their derivatives ...................

A. P. Morse and Donald Chesley Pfaff, Separative relations for

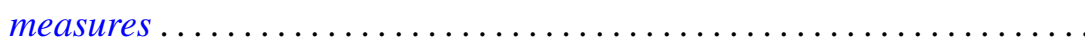

Albert David Polimeni, Groups in which $\operatorname{Aut}(G)$ is transitive on the

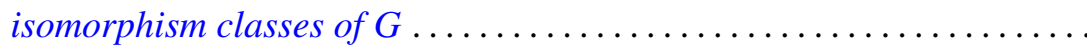

Aribindi Satyanarayan Rao, Matrix summability of a class of derived

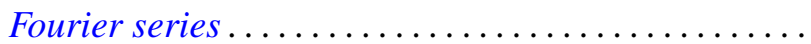

Thomas Jay Sanders, Shape groups and products

Ruth Silverman, Decomposition of plane convex sets. II. Sets associated

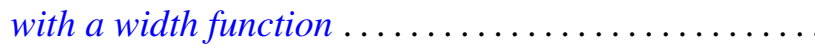

Richard Snay, Decompositions of $E^{3}$ into points and countably many flexible dendrites.............................

John Griggs Thompson, Nonsolvable finite groups all of whose local subgroups are solvable, IV ...

Robert E. Waterman, Invariant subspaces, similarity and isometric equivalence of certain commuting operators in $L_{p} \ldots$

James Chin-Sze Wong, An ergodic property of locally compact amenable

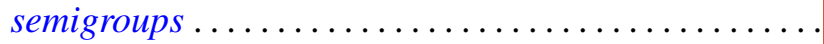

Julius Martin Zelmanowitz, Orders in simple Artinian rings are strongly equivalent to matrix rings ....................... 